\title{
HALVING CLOSED CURVES IN NORMED PLANES AND RELATED INEQUALITIES
}

\author{
Chan He, Horst Martini and Senlin Wu
}

\begin{abstract}
In an arbitrary normed plane we study the relation between the length of a closed curve and the length of its midpoint curve as well as the length of its image under the halving pair transformation. We show that the image curve under the halving pair transformation is convex provided the original curve is convex. We give a sufficient condition for the geometric dilation of a closed convex curve to be larger than a quarter of the perimeter of the unit circle. Moreover, we obtain several inequalities to show the relation between the halving distance and other quantities well known in convex geometry.
\end{abstract}

Mathematics subject classification (2000): 52A21, 52A10, 46C15.

Keywords and phrases: Arc length, Birkhoff orthogonality, circumradius, convex curve, convex geometry, detour, geometric dilation, geometric inequality, halving pair, halving distance, inradius, midpoint curve, minimum width, Minkowski plane, normed plane.

\section{REFERENCES}

[1] G. Aver Kov, On cross-section measures in Minkowski spaces, Extracta Math., 18 (2003), 201-208.

[2] G. BIR KHOFF, Orthogonality in linear metric spaces, Duke Math. J., 1 (1935), 169-172.

[3] A. Dumitrescu, A. Ebbers-Baumann, A. Grüne, R. Klein, G. Rote, On the geometric dilation of closed curves, graphs, and point sets, Comput. Geom., 36 (2007), 16-38.

[4] A. Ebbers-Baumann, A. Grüne, R. Klein, Geometric dilation of closed planar curves: New lower bounds, Comput. Geom., 37 (2007), 188-208.

[5] A. GRÜNE, Geometric dilation and halving distance, Ph.D. Dissertation, Universität Bonn. 2006.

[6] R. C. JAMES, Orthogonality and linear functionals in normed linear spaces, Trans. Amer. Math. Soc., 61 (1947), 265-292.

[7] H. Martini, K. J. Swanepoel, The geometry of Minkowski spaces - a survey. Part II, Expositiones Math., 22 (2004), 93-144.

[8] H. Martini, K. J. Swanepoel, Antinorms and Radon curves, Aequationes Math., 72 (2006), 110 138.

[9] H. Martini, K. J. Swanepoel, G. Weiss, The geometry of Minkowski spaces - a survey. Part I, Expositiones Math., 19 (2001), 97-142.

[10] H. Martini, S. Wu, Geometric dilation of closed curves in normed planes, Comput. Geom., to appear.

[11] A. C. Thompson, Minkowski Geometry, Encyclopedia of Mathematics and Its Applications, Vol. 63, Cambridge University Press, Cambridge, 1996. 